\title{
Natural melanogenesis stimulator a potential tool for the treatment of hypopigmentation disease
}

\begin{abstract}
The synthesis of melanin pigments or melanogenesis has many important physiological functions that include photo protection of the human skin from ultraviolet (UV) irradiation. Melanogenesis is a complex pathway involving melanin synthesis, melanin transport, and melanosome release. Melanin synthesis is stimulated by various effects such as $\alpha$-melanocyte- stimulating hormone $(\alpha-\mathrm{MSH})$; cyclic AMP (cAMP) elevating agents including palmitic acid, methyl ester, psoralen, thymoquinone, piperine, berberine and withaferin-A. Loss of the hair shaft melanin is associated with decrease of tyrosinase activity in the bulb of melanocytes. Activators of tyrosinase with stimulatory effects on melanogenesis are beneficial for the treatment of hypopigmentation diseases. This is only report authors found in literature about tyrosinase activation. Melanogenesis stimulator are rarely studied as most industries are more interested on inhibitors of the enzyme in order to reduce the adverse effects of melanin formation in, for example, processed food and human skin. Hence, there is a need to review the successful utilization of tyrosinase stimulators from natural herbs for medicinal applications.
\end{abstract}

Volume 2 Issue I - 2017

\author{
Kamal Uddin Zaidi,' Sharique A Ali, ${ }^{2}$ Ayesha S \\ Ali, ${ }^{2}$ Vijay Thawani' \\ 'Biotechnology Pharmacology Laboratory CSRD, Peoples \\ University, India \\ ${ }^{2}$ Postgraduate Department of Biotechnology and Zoology Saifia \\ College of Science, India
}

\author{
Correspondence: Kamal Uddin Zaidi Author name, \\ Biotechnology Pharmacology Laboratory CSRD, Peoples \\ University, Bhopal, (M.P) 462037, India, \\ Email Zaidi.kamal92@gmail.com
}

Received: October 27, 2016 | Published: February 24, 2017

Keywords: tyrosinase, herbs, vitiligo, melanin, melanocytes

Abbreviations: $\alpha$-MSH, $\alpha$-melanocyte- stimulating hormone; cAMP, cyclic adenosine monophosphate; MITF, microphthalmiaassociated transcription factor; TRP1, tyrosinase related protein1; TRP2,tyrosin aserelated protein2; CREB, response element-binding protein; mRNA, messenger ribonucleic acid

\section{Introduction}

Vitiligo is one of the most well-known conditions of skin depigmentation. It is estimated to occur in up to $2 \%$ of the world's population. A number of people, these patches can prove all over the body. It is an autoimmune disease in which the pigment-inducing cells are injured. There is no therapy for vitiligo, however there are few therapies, including ultraviolet light, cosmetic cover-ups or corticosteroid creams medications. ${ }^{2}$ Melanin synthesis is regulated by melanocyte specific enzymes and related transcription factors, tyrosinase, TRP-1, TRP-2 and MITF. ${ }^{1}$ Natural herbal extracts have powerful phytochemical properties which are now being exploited world over and there is a sudden surge in Ayurvedic or traditional uses of plant wealth in treatment of diseases like cancers, arthritis, sterility, psoriasis and diabetes. For the treatment of hypopigmentation or vitiligo, plant extracts have been used since time immemorial. ${ }^{3}$ However, still, there are no conclusive data available with regard to various tyrosinase stimulators on the mechanism of melanogenesis in different vertebrate species including human beings and reptiles. It is not clearly known that whether plant extracts induce increase arborisation of melanocyte dendrites or increase the number of melanin granules, or work through tyrosinase or any other signal transduction pathway in reactivating melanin polymerization within the pigment cells melanophores or melanocytes involving cellular receptor activation. We focus our attention on the use of melanogenesis stimulators from natural herbs. From the present review, it is become clear that the plant extracts play a major role against various hypopigmentary anomalies and this would provide an experimental basis for development of new pharmacotherapy's against dermatological anomalies like treatment of vitiligo.

\section{Effect of herbal extract and their actives on melanogenesis}

Herbal extracts most widely used medicinal plants in traditional oriental medicine. Over thousands of years, it has been used to improve the overall condition of skin, as well as to treat a wide variety of diseases. Natural plant extracts have powerful properties which are now being exploited world over and there is a sudden surge in Ayurvedic or traditional uses of plant wealth in treatment of diseases like cancers, arthritis, sterility, psoriasis and diabetes. For the treatment of hypopigmentation or vitiligo. Kyoko et al. ${ }^{4}$ stated whether Tunisian aromatic plants can induce melanogenesis in aromatic plant extracts; found that melanogenesis in the cultured mouse B16 melanoma cell line was enhanced by Tunisian aromatic plants. The cells cultured with and without Tunisian plant extracts showed no effect on cell growth and shape. This denotes that Tunisian aromatic plants can induce melanogenesis in B16 melanoma cells without causing transformation. Later on Jeon ${ }^{5}$ studied the essential oil from lotus flower extract and its effects on melanogenesis in human melanocytes. It was found that the effective compound of lotus flower oil palmitic acid methyl ester induced the expression of tyrosinase, microphthalmia- associated transcription factor M (MITF-M), and tyrosinase-related proten-2 (TRP-2) proteins, but not tyrosinase mRNA. As the result of increased tyrosinase expression, thereby indicating that it may play a role in the regulation of melanin content. In 2011 Ali and co-workers determined the effect of lyophilized extracts of Psoralea corylifolia seeds extract on the isolated scale melanophores of Channa punctatus. Authors found that the lyophilized extract of P. Corylifolia and its active ingredient psoralen caused significant melanin dispersal responses leading to darkening of the fish scale melanophores, which were completely antagonized by atropine and hyoscine. These melanin dispersal effects were also found to be markedly potentiated by neostigmine, an anti cholinesterase agent. ${ }^{6}$ Ali \& Meitei $^{7}$ also studied the effects of the lyophilized seed extract of Nigella sativa and its active ingredient, thymoquinone on the isolated melanophores of the wall lizard to find the mechanism of skin darkening at the cellular 
level. The pigment cells responded by distinct dispersion leading to skin darkening. The effect was physiologically significant as reimmersion in physiological saline made the melanophores return to their normal intermediate state.

Lyophilized dried fruit extracts of Piper nigrum and pure piperine on the tadpole melanophores of frog Rana tigerina which offered excellent In vitro opportunities for studying the effects of pharmacological and pharmaceutical agents. The nature of specific cellular receptors present on the neuro-melanophore junction and their involvement in pigmentary responses has been explored. The extract of $P$. Nigrum and its active ingredient piperine caused significant melanin dispersal responses leading to darkening of the tail melanophores, which were completely antagonized by atropine and hyoscine. These per se melanin dispersal effects were also found to be markedly potentiated by neostigmine an anti cholinesterase agent. According to theses workers, it appeared that the melanin dispersal effects of the extracts of P. Nigrum and pure piperine leading to skin darkening are mediated by cholinergic muscarinic or piperine-like receptors having similar properties. ${ }^{8}$ Root extracts of Withania somnifera along with pure withaferin-A, on the isolated skin melanophores of frog, Rana tigerina which are disguised type of smooth muscle cells and offer excellent In vitro opportunities for studying the effects of pharmacological and pharmaceutical agents. The lyophilized extract of $W$. Somnifera and its active ingredient withaferin-A induced powerful dose dependent physiologically significant melanin dispersal effects in the isolated skin melanophores of $R$. Tigerina, which were completely blocked by atropine as well as hyoscine. The per se melanin dispersal effects of lyophilized extracts of $W$. Somnifera and its active ingredient withaferin-A got highly potentiated by neostigmine. It appears that the melanin dispersal effects of the extracts of $W$. Somnifera and withaferin-A is mediated by cholino-muscarinic like receptors having similar properties. ${ }^{9}$

In vitiligo the active melanocytes in the epidermis are totally missing, whereas melanoblast cells in the outer root sheath of hair follicles are not affected. In an attempt to find potent repigmenting agents for vitiligo therapy, pod extracts of Cassia occidentalis were found to be effective in inducing differentiation and migration of mouse melanoblast cell lines. The induction in melan-a melanoblast cells after 4 days in treatment medium. In addition it induced the tyrosinase activity and altered melb-a cell morphology. Trans well migration assay showed the potential of this herbal candidate to induce direct migration of treated cells. The findings were significant in designing preclinical and clinical studies on the efficacy of $C$. Occidentalis as a stimulant for skin regimentation in vitiligo. ${ }^{10}$ Moreira et al. ${ }^{11}$ investigated the melanogenic activity of hydro alcoholic extracts from the leaves and flowers of $P$. Venusta on murine B16F10 melanoma cells. Both extracts, leaves increased the melanin content in a dose dependent manner on melanoma cells. Leaves extract promoted enhancement of melanogenesis with maximum effect of $(3 \mu \mathrm{g} / \mathrm{mL})$, and the flower extract increased in $(0.1 \mu \mathrm{g} / \mathrm{mL})$. The cell viability tested concentrations of both extracts no cell death was detected. Actually, either extract was not able to cause any change in the tyrosinase activity. Their findings support the folk medicinal use of $P$. Venusta on the treatment of hypopigmentation diseases, such as vitiligo Table 1.

Table I Natural melanogenesis stimulator

\begin{tabular}{|c|c|c|c|c|}
\hline Source & Bioactive & In vitro /In vivo & Target site/ Mode of action & References \\
\hline Agaricus bisporus & Tyrosinase & BI6FI0 Melanocytes & Phosphorylation cAMP and CREB & Zaidi et al., ${ }^{18}$ \\
\hline Pleurotus ostreatus & Tyrosinase & BI6FI0 Melanocytes & TRPI,TRP2 and MITF & Zaidi et al., ${ }^{17}$ \\
\hline Tunisian aromatic plants & Plants extracts & BI6 melanoma cell line & Tyrosinase TRPI,TRP2 & Kyoko et al., ${ }^{4}$ \\
\hline Nelumbo nuficera & Palmitic acid Methyl ester & Human melanocytes & MITF,TRP-2 & Jeon $^{5}$ \\
\hline Psoralea corylifolia & Psoralen & Fish scale Melanophores & Melanin dispersal responses & Ali et al., ${ }^{6}$ \\
\hline Nigella sativa & Thymoquinone & Wall lizard of melanophores & Melanin dispersal responses & Ali \& Meitei, ${ }^{7}$ \\
\hline Piper nigrum & Piperine & $\begin{array}{l}\text { Tadpole Melanophores of } \\
\text { frog Rana tigerina }\end{array}$ & Melanin dispersal responses & Sajid \& Ali, ${ }^{8}$ \\
\hline Withania somnifera & Withaferin-A & Melanophores of frog & Melanin dispersal effects & Ali \& Meitei, ${ }^{7}$ \\
\hline Cassia occidentalis & Pod extracts & Melanoblast cell lines & Regimentation in vitiligo & Babitha et al. ${ }^{10}$ \\
\hline Ficus carica & Psoralen & Reptilian melanophores & Melanin dispersal effects & Ali \& Meitei ${ }^{7}$ \\
\hline $\begin{array}{l}\text { Helminthostachys } \\
\text { zeylanica }\end{array}$ & $\begin{array}{l}\text { 4'-O- } \beta \text {-d-Glucopyranosyl- } \\
\text { quercetin-3-O- } \beta \text {-d- } \\
\text { glucopyranosyl- }(I \rightarrow 4)-\beta-d \\
\text { glucopyra-noside }\end{array}$ & BI6 melanoma cells & Intracellular melanogenesis & $\begin{array}{l}\text { Yamauchi et } \\
\text { al. }^{.15}\end{array}$ \\
\hline Berberis vulgaris, & Berberine & Melanophores of toad & Melanin dispersion response & Ali et al. ${ }^{6}$ \\
\hline Agaricus bisporus & Tyrosinase & BI6FI0 Melanocytes & Phosphorylation cAMP and CREB & Zaidi et al. ${ }^{17}$ \\
\hline
\end{tabular}

a-MSH, a-melanocyte- stimulating hormone.

Camp, cyclic adenosine monophosphate.

MITF, Microphthalmia-associated transcription factor.

TRPI, Tyrosinase related protein I.

TRP2, Tyrosinase related protein 2.

CREB, Response element-binding protein.

Citation: Zaidi KU,Ali SA, Ali A, et al. Natural melanogenesis stimulator a potential tool for the treatment of hypopigmentation disease. Int J Mol Biol Open Access. 20I7;2(I):37-40. DOI: 10.15406/ijmboa.2017.02.000I2 
Later on Ali \& Meitei ${ }^{12}$ studied the effects of the root extract of Withania somnifera and its active ingredient Withaferin A on the isolated melanophores of the wall lizard $H$. Flaviviridis were studied in order to establish the mechanism of skin darkening at the cellular level. Significant skin darkening activity of the extract of $W$. Somnifera and Withaferin A was observed on the isolated melanophores of the wall lizard. The melanin dispersal effects leading to the darkening of the skin were antagonized by atropine and hyoscine, and were also found to be highly potentiated by neostigmine. These findings suggested that the extract of $W$. Somnifera, as well as its active principle, mimic the action of acetylcholine in melanin dispersion, thus leading to skin darkening via stimulation of cholinergic receptors of muscarinic nature within the melanophores of the wall lizard. In 2012, Meitei \& $\mathrm{Ali}^{13}$ subsequently studied the effects of fig leaf extract and its bioactive compounds which were found to induce skin darkening effect in reptilian melanophores via cholinergic receptor stimulation. They found ethanolic leaf extract of Ficus carica per se can cause melanin stimulatory effects leading to skin darkening. They also found neostigmine an anti cholinesterase agent to potentiate the melanin dispersal effects of both ethanolic leaf extract of F. Carica and its active ingredient psoralen. Choudhary et al. ${ }^{14}$ investigated effects of extracts of two species of Chlorophytum i.e., Chlorophytum tuberosum \& Chlorophytum borivilianum on the isolated scale melanophores of the teleost fish, Channa punctatus. The lyophilized extract of tubers of $C$. Tuberosum had a melanin aggregating effect causing paling of the skin; the action seems to be mediated through alpha adrenergic receptors present dominantly on fish melanophores. The extract of tubers of $C$. Borivilianum had a melanin dispersing effect within the fish melanophores inducing darkening of the skin and the responses seem to be mediated probably through beta adrenergic receptors.

The compound 4'-O- $\beta$-d-Glucopyranosyl-quercetin-3-O- $\beta$-dglucopyranosyl-( $1 \rightarrow 4)-\beta$-d glucopyra-noside from Helminthostachys zeylanica root extract as a melanogenesis acceleration compound and have synthesized using rutin as the starting material. It has been found that isolated compounds were also synthesized to understand the structure-activity relationships in melanin biosynthesis. Melanogenesis activities of the glycosides were determined by measuring intracellular melanin content in B16 melanoma cells. Among the synthesized quercetin glycosides, quercetin-3-O- $\beta$-d-glucopyranoside quercetin3 -O- $\beta$-d-glucopyranosyl-( $(1 \rightarrow 4)-\beta$-d-glucopyranoside, and 3 showed more potent intracellular melanogenesis acceleration activities than theophyline used as positive control in a dose-dependent manner with no cytotoxic effect. ${ }^{15}$ Ali et al. ${ }^{16}$ have also determined the ability of berberine, a principal active ingredient present in the roots of the herb Berberis vulgaris, to stimulate pigment dispersion in the isolated skin melanophores of the toad Bufo melanostictus. It was observed for mean melanophore size index of the isolated skin melanophores of $B$. Melanostictus and assayed after treating with various concentrations of berberine. A marked melanin dispersion response leading to skin darkening was observed in the isolated melanophores of toad in response to berberine, which was found to be mediated through beta- 2 adrenergic receptors. It was indicated that berberine causes a tremendous, dose-dependent, physiologically significant pigment dispersing in the isolated skin melanophores of B. Melanostictus.

Recently Zaidi el al. ${ }^{17}$ has been investigated the effect of purified mushroom tyrosinase of Agaricus bisporus on B16F10 melanocytes for the melanin production via blocking pigment cell machinery. Using B16F10 melanocytes showed that the stimulation of melanogenesis by purified tyrosinase is due to increased tyrosinase absorption. Cellular tyrosinase activity and melanin content in B16F10 melanocytes were increased by purified tyrosinase in a dose-dependent manner. Western blot analysis revealed that cellular tyrosinase levels were enhanced after treatment with purified tyrosinase for 48hours. Furthermore, tyrosinase induced phosphorylation of cyclic adenosine monophosphate (cAMP) response element-binding protein (CREB) in a dose-dependent manner. The purified tyrosinase-mediated increase of tyrosinase activity was significantly attenuated by H89, LY294002, Ro-32-0432, and PD98059, cAMP-dependent protein kinase inhibitors. The results indicate that purified tyrosinase can be used as contestant for the treatment of vitiligous skin conditions. Later Zaidi el al. ${ }^{18}$ also reported that morphoantomical effects of purified tyrosinase to determine its skin-darkening potential using B16F10 melanocyte. Phase contrast and immuno fluorescence microscopic analysis of B16F10 melanocytes has been done after treatment with various concentrations of purified tyrosinase along with standard tyrosinase (Sigma) in order to explore the mechanism of action of purified tyrosinase induced skin darkening. The phase contrast microscopic results showed that the number of melanocytes with melanin-loaded dendrites has increased significantly in purified tyrosinase treated cells in a dose dependent manner leading to skin darkening. In addition, immuno fluorescence microscopic analysis revealed purified tyrosinase increase cellular tyrosinase expression in doze dependent manner due to tyrosinase absorption in B16F10 melanocyte. Present findings proved that purified tyrosinase possesses a skin darkening potential and could be used as a safe melanogenic agent for the treatment of hypopigmentation disorders or vitiligo.

\section{Conclusion}

Different dermatological disorders, such as vitiligo, albinism and loss of the hair, the tyrosinase stimulators induce cellular melanin biosynthesis, up-regulating CREB phosphorylation and expression of MITF, tyrosinase, TRP-1 and TRP-2, and tyrosinase. These consistent results suggest that melanogenesis stimulators might be useful for treatment of hypopigmentation related disorders. The exploration and characterization of new stimulators of tyrosinase are not only useful for the medicinal purposes, but their potential applications in improving food quality and nutritional value, controlling insect pests etc are also important.

\section{Acknowledgements}

The authors are grateful to People's University, Bhopal, for providing laboratory facilities and for granting financial assistance to carry out this work, and Principal and Secretary, Saifia College of Science, Bhopal, for encouragement.

\section{Conflict of interest}

Author declares that there is no conflict of interest.

\section{References}

1. Tuerxuntayi A, Yong-qiang Liu, Ablajan Tulake, et al. Kaliziri extract up regulates tyrosinase, TRP-1, TRP-2 and MITF expression in murine B16 melanoma cells. BMC Complement Altern Med. 2014;14:166.

2. Sanju Arianayagam, Terence J Ryan. Disorders of pigmentation of the skin-hypotheses underlying interventions by multiple systems of medicine: is there a role for integrated medicine? Current Sci. $2016 ; 111(2): 325-336$. 
3. Ali AS, Naaz I, Choudhary R. Berberine-induced pigment dispersion in Bufo melanostictus melanophores by stimulation of beta-2 adrenergic receptors. J Recept Sig Trans Res. 2014;34(1):15-20.

4. Kyoko M, Mitsuko K, Kchouk SH, et al. Effect of Tunisian Plant Extract on Melanogenesis. Animal Cell Technology:Basic Applied Aspects. 2009;15:259-264.

5. Jeon S, Kim NH, Koo BS, et al. Lotus (Nelumbo nuficera) flower essential oil increased melanogenesis in normal human melanocytes. $J$ Exp Mol Med. 2009;41(7):517-524.

6. Ali AS, Sultan T, Galgut J, et al. In vitro responses of fish melanophores to lyophilized extracts of Psoralea corylifolia seeds and pure psoralen. Pharm Biol. 2011;49(4):422-427.

7. Ali AS, Meitei V. Nigella sativa seed extract and its bioactive compound thymoquinone on the new melanogens causing hyper pigmentation in the wall lizard melanophores. J Pharm Pharmacol. 2011;63(5):741-746.

8. Sajid M, Ali AS. Mediation of cholino-piperine like receptors by extracts of Piper nigrum induces melanin dispersion in Rana tigerina tadpole melanophores. J Recept Sig Trans. 2011;31(4):286-290.

9. Ali AS, Meitei V. On the action and mechanism of withaferin-A from Withania somnifera, a novel and potent melanin dispersing agent in frog melanophores. J Recept Sig Transduct Res. 2011;31(5):359-366.

10. Babitha S, Shin JH, Nguyen DH, et al. A stimulatory effect of Cassia occidentalis on melanoblast differentiation and migration. Arch. Dermatol. Res. 2011;303(3):211-216.
11. Moreira CG, Horinouchi CD, Filho CS, et al. Hyperpigmentant activity of leaves and flowers extracts of Pyrostegia venusta on murine B16F10 melanoma. J Ethnopharmacol. 2012;141(3):1005-10011.

12. Ali AS Meitei V. Withania somnifera root extracts induce skin darkening in wall lizard melanophores via stimulation of cholinergic receptors. Nat Prod Res. 2012;26(17):1645-1648.

13. Meitei KV, Ali AS. Fig leaf extract and its bioactive compound psoralen induces skin darkening effect in reptilian melanophores via cholinergic receptor stimulation In Vitro. Cell Dev Biol Animal. 2012;48:335-339.

14. Chaudhari SA, Peter J, Galgut JM, et al. Melanin inhibitory and melanin stimulatory effects of extracts of Chlorophytum tuberosum and Chlorophytum borivilianum on isolated fish scale melanophores. African J Pharmacy and Pharmacol. 2012;6(12):919-923.

15. Yamauchi K, Mitsunaga T. Batubara I Synthesis of quercetin glycosides and their melanogenesis stimulatory activity in B16 melanoma cells. Bioorganic Med Chem. 2014;22(3):937-944.

16. Ali AS, Naaz I, Choudhary R. Berberine-induced pigment dispersion in Bufo melanostictus melanophores by stimulation of beta- 2 adrenergic receptors. J Recept Sig Trans Res. 2014;34(1):15-20.

17. Zaidi KU, Ali AS, Ali SA. Effect of Purified Mushroom Tyrosinase on Melanin Content and Melanogenic Protein Expression. Biotechnol Res Int. 2016;2016:1-8.

18. Zaidi KU, Ali AS, Ali SA. Melanogenic effect of purified mushroom tyrosinase onB16F10 Melanocytes: A Phase Contrast and Immuno fluorescence Microscopic Study. J Microsc Ultrastructure. 2016;5:1-6. 